\title{
Estoma \& Câncer Retal: Revisão de 195 Estomas Realizados em 380 Pacientes Portadores de Câncer Retal
}

\author{
Stoma and Rectal Cancer: Review of 195 Stomas Carried out in 380 Patients \\ with Rectal Cancer
}

\author{
GERALDO MAGELAGOMES DACRUZ1, MÔNICAMOURTHÉ DEALVIM ANDRADE², DANIEL MARTINS BARBOSA \\ MEDEIROS GOMES ${ }^{2}$, JOSÉ ROBERTO MONTEIRO CONSTANTINO ${ }^{2}$, BRUNOCUNHACHAMONE ${ }^{2}$
} ${ }^{1}$ Mestre, Doutor e Professor Titular de Coloproctologia, TSBCP, TFBG, TALACP, TCBC, TISUCRS, FASCRS; ${ }^{2}$ Pós-
graduandos lato sensu em Coloproctologia (residência e especialização).

CRUZ GMG; ANDRADE MMA; GOMES DMBM; CONSTANTINO JRM; CHAMONE BC. Estoma \& Câncer Retal: Revisão de 195 Estomas Realizados em 380 Pacientes Portadores de Câncer Retal. Rev bras Coloproct, 2008;28(2): 193-203.

RESUMO: Em uma casuística de 24.000 pacientes 923 eram portadores de tumores de intestino grosso (3.8\%), 870 dos quais eram tumores colorretais $(\mathbf{9 4 , 2} \%)$, dos quais 490 eram câncer no cólon $(53,1 \%)$ e 380 no reto $(41,2 \%)$ e apenas 53 tumores anais $(5,7 \%)$. $O$ objetivo deste trabalho é estudar 380 pacientes portadores de câncer retal, analisando, especificamente, os 195 estomas criados nos mesmos, estratificando-os em temporários e definitivos, descrevendo suas modalidades e indicações bem como suas complicações e abordagem cirúrgicas das mesmas. De 380 pacientes portadores de câncer retal 373 foram operados $(98,2 \%)$ e 338 tiveram os tumores removidos $(\mathbf{9 1 , 8 \%})$, tendo sido a retossigmoidectomia abdominal a técnica cirúrgica mais realizada (172 ou 45,3\%), das quais, 133 com anastomose manual $(35,0 \%)$ e 39 com anastomose mecânica (10,3\%), seguida pela amputação abdominoperineal (135 casos ou $35,5 \%)$. Dos 373 pacientes operados foram realizados estomas em 195 (52,3\%), 174 dos quais definitivos $(46,6 \%)$ e 21 temporários (5,7\%). Destarte, dos 195 estomas realizados, 174 foram definitivos $(\mathbf{8 9 , 2 \% )}$ e 21 temporários (10,8\%). As modalidades de estomas mais realizados foram colostomias terminais (146 casos, 39,1\%) e colostomias em alça (30 casos, 8,0\%). Dos 21 estomas temporários nove foram colostomias em alça (5,2\%) e 12 foram ileostomias em alça (7,0\%). Em 16 dos 21 casos o estoma foi feito como protetor de anastomose em 133 casos de ressecção com anastomose manual (oito colostomias em alça e oito ileostomias em alça); e cinco foram realizados em 39 casos de anastomoses mecânicas, sendo uma colostomia em alça e quatro ileostomias em alça. Os 174 estomas definitivos foram feitos em 135 casos de amputação abdominoperineal, 35 em tumores irressecáveis e quatro em proctocolectomia, tendo sido 146 colostomias terminais $(83,9 \%)$, quatro ileostomias terminais $(2,3 \%), 21$ colostomias em alça $(12,1 \%)$ e três colostomias duplas $(1,7 \%)$. Ocorreram 14 complicações $(7,2 \%)$ nos 195 estomas executados, assim distribuídas: oito complicações em 146 colostomias terminais $(5,5 \%)$, uma complicação dentre as 30 colostomias em alça (3,3\%), três complicação nas três colostomias duplas $(100,0 \%)$, duas complicações em 12 ileostomias am alça $(16,7 \%)$ e nenhuma complicação entre as quatro ileostomias terminais. Estenose e prolapso foram as complicações mais comuns (quatro casos de cada).

Descritores: Estomas; câncer retal; estoma em câncer retal.

\section{OBJETIVO}

O objetivo deste trabalho é estudar 380 pacientes portadores de câncer retal, analisando, especificamente, os 195 estomas criados nos mesmos, estratificando-os em temporários e definitivos, descrevendo suas modalidades e indicações bem como suas complicações e abordagem das mesmas. São analisados os dados relacionados às várias cirurgias a que os pacientes foram submetidos, tanto como casos eletivos quanto como de urgência, estudando os que receberam estomas, seja como opção cirúrgica em cirurgias eletivas (e.g., estomas protetoras de anastomoses), ou opção de conduta em cirurgias de urgência (impossibi- 
Rev bras Coloproct Abril/Junho, 2008
Estoma \& Câncer Retal: Revisão de 195 Estomas Realizados em 380 Pacientes Portadores de Câncer Retal Geraldo Magela Gomes da Cruz e Cols.
Vol. 28 lidade de anastomose imediata) ou mesmo como alternativa cirúrgica única (pacientes com tumores irressecáveis).

\section{MATERIAL E MÉTODO}

O material provém de um fichário contendo 24.000 pacientes, dos quais 870 eram portadores de câncer colorretal, diagnosticados como colônicos (490 casos) e retais (380 casos). Foram realizadas 370 cirurgias nos 380 pacientes, nos quais foram confeccionados 195 estomas. A casuística analisada constitui-se desses 194 pacientes que receberam estomas como opção cirúrgica, quer em forma de estomas temporários quer em forma de estomas definitivos.

\section{RESULTADOS}

Incidência de câncer no intestino grosso estratificados pelos cólons, reto e ânus:

Houve 490 casos de tumores localizados nos cólons $(53,1 \%)$, seguidos pelos tumores de reto (380 casos, $41,2 \%)$ e apenas 53 tumores $(5,7 \%)$ localizados no ânus e canal anal (tabela 1 e figura 1).

Pacientes operados, tumores ressecados e cirurgias realizadas em 380 pacientes portadores de câncer retal:

Dos 380 pacientes portadores de câncer retal sete não chegaram a ser operados em decorrência da gravidade dos mesmos $(1,8 \%)$ (tabela 2 e figura 2 ). Dos 373 pacientes operados 35 Não tiveram os tumores ressecados $(9,2 \%)$. E dos 338 pacientes que tiveram os tumores ressecados $(88,9 \%)$, foram as seguintes as técnicas cirúrgicas realizadas: a cirurgia mais realizada (tabela 2 e figura 2) foi a amputação abdominoperineal, em 135 pacientes (35,5\%). A ressecção local foi realizada em 27 pacientes $(7,1 \%)$. A retossigmoidectomia abdominal com anastomose colorretal manual foi realizada em 104 pacientes (27,4\%); a retossigmoidectomia abdomino-endoanal com anastomose colorretal manual em 22 pacientes $(5,8 \%)$; e a retocolectomia total com anastomose ileorretal manual em 7 pacientes $(1,8 \%)$, totalizando 133 casos de anastomoses manuais $(35,0 \%)$. A retossigmoidectomia abdominal com anastomose colorretal mecânica foi realizada em 26 pacientes $(6,9 \%)$ e a retocolectomia total com anastomose coloanal mecânica em 13 pacientes $(3,4 \%)$, totalizando 39 casos de anastomoses mecânicas (10,3\%) (tabela 2 e figura 2).

Sinopse de pacientes operados, de tumores ressecados e cirurgias básicas realizadas em 380 pacientes portadores de câncer retal:

Dos 380 pacientes portadores de câncer retal, sete não chegaram a ser operados em decorrência da gravidade dos mesmos (1,9\%); e dos 373 pacientes

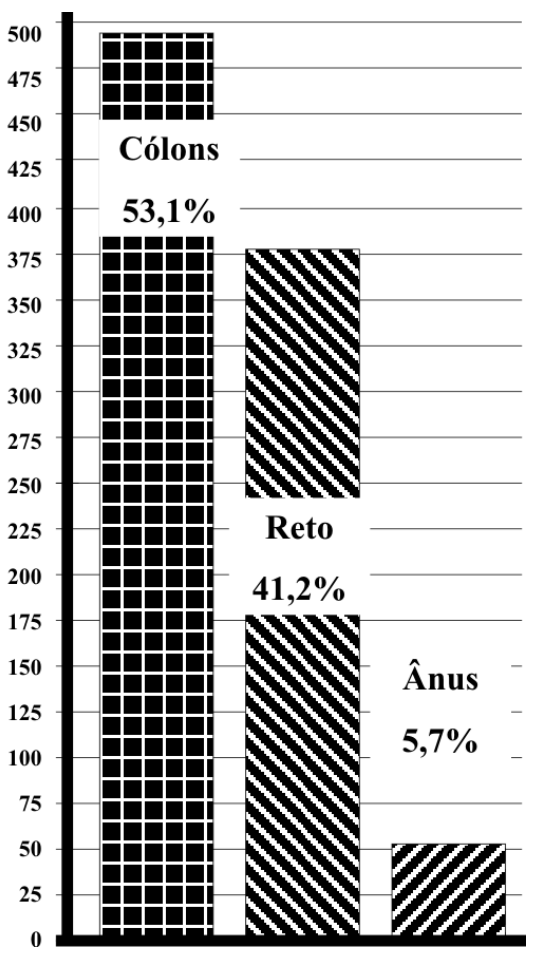

Figura 1 - Distribuição de 973 tumores anocolorretais.

Tabela 1 - Distribuição de 923 tumores malignos nos cólons, reto e ânus.

\begin{tabular}{lrrc}
\hline Distribuição de Tumores Malignos no IG & N & \% & Soma \\
\hline Cólons & 490 & $53,1 \%$ & 53,1 \\
Reto & 380 & $41,2 \%$ & $94,3 \%$ \\
Ânus e canal anal & 53 & $5,7 \%$ & $100,0 \%$ \\
Total & $\mathbf{9 2 3}$ & $\mathbf{1 0 0 , 0 \%}$ & \\
\hline
\end{tabular}


Tabela 2 - Sinopse das 373 cirurgias realizadas em 380 pacientes portadores de câncer retal.

\begin{tabular}{lrc}
\hline Cirurgias & $\mathbf{N}^{\mathbf{0}}$ & $\mathbf{\%}$ \\
Ressecções locais (RL) & $\mathbf{2 7}$ & $\mathbf{7 , 1}$ \\
RSA-ACRMa & 104 & 27,4 \\
RCT-AIRMa & 22 & 5,8 \\
RSAEA-ACRMa & 7 & 1,8 \\
Anastomoses manuais & $\mathbf{1 3 3}$ & $\mathbf{3 5 , 0}$ \\
RSA-ACRMe & 26 & 6,9 \\
RCT-ACAMe & 13 & 3,4 \\
Anastomoses mecânicas & $\mathbf{3 9}$ & $\mathbf{1 0 , 3}$ \\
AAP - MILES & 135 & 35,5 \\
PCT & 4 & 1,0 \\
Amputação sem e com colectomia total & $\mathbf{1 3 9}$ & $\mathbf{3 6 , 5}$ \\
TU ressecados & 338 & 88,9 \\
& & \\
Total de pacientes operados & 373 & 98,1 \\
Total de pacientes não operados & 7 & 1,9 \\
Total de pacientes com CR & 380 & 100,0 \\
Total de TU ressecados & & 88,9 \\
Total de TU irressecáveis - colostomia & 338 & 9,12 \\
Total de pacientes com CR & 35 & 100,0 \\
\hline
\end{tabular}

RL: resseção local;

RSA-ACRMa: retossigmoidectomia abdominal com anastomose colorretal manual;

RCT-AIRMa: retocolectomia total com anastomose ileorretal manual;

RSAEA-ACRMa: retossigmoidectomia abdômino-endoanal com anastomose colorretal manual;

RSA-ACRMe: retossigmoidectomia abdominal com anastomose colorretal mecânica;

RCT-ACAMe: retocolectomia com anastomose cólon-anal mecânica;

AAP: amputação abdominoperineal à Miles com colostomia definiiva;

PCT: proctocolectomia total.

operados $(98,1 \%) 35$ não tiveram os tumores ressecados $(9,2 \%)$. E dos 338 pacientes que tiveram os tumores ressecados 27 foram submetidos à ressecção local $(7,1 \%), 139$ à amputação abdominoperineal $(36,5 \%)$ e 172 à ressecções intestinais, dos quais 133 à ressecções com anastomoses manuais $(35,0 \%)$ e 39 à ressecções com anastomoses mecânicas $(10,3 \%)$ (tabela 3 e figura 3).

Estomas confeccionados nas várias cirurgias para abordagem de 380 pacientes portadores de câncer no reto:

$\mathrm{Na}$ abordagem de 380 pacientes portadores de câncer nos cólons, 7 não chegaram a ser operados, sendo que, dos 373 operados $195(52,3 \%)$ receberam algum tipo de estoma, sendo $174(46,6 \%)$ definitivos e 21 temporários $(5,6 \%)$. Os 21 estomas temporários foram feitos em 16 dos 133 pacientes submetidos a

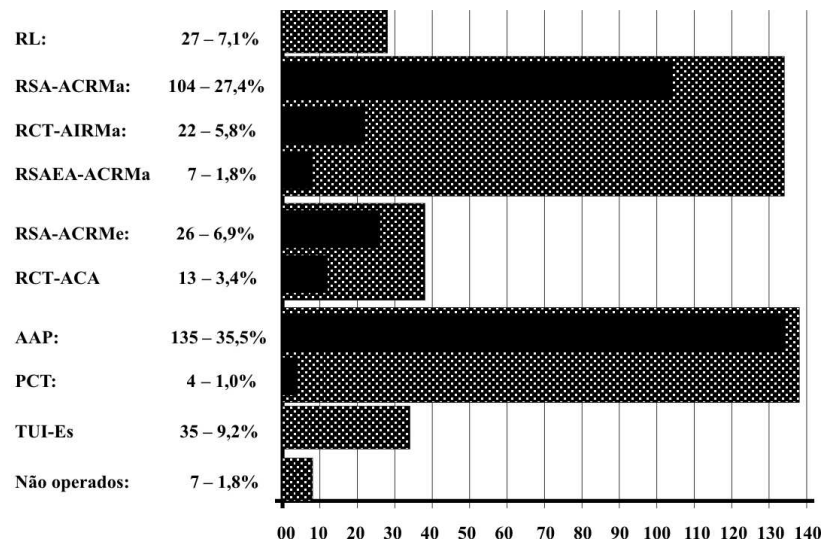

Figura 2 - Sinopse das incidências das 373 cirurgias realizadas em 380 pacientes portadores de câncer retal.

RL: resseção local; RSA-ACRMa: retossigmoidectomia abdominal com anastomose colorretal manual; RCT-AIRMa: retocolectomia total com anastomose ileorretal manual; RSAEA-ACRMa: retossigmoidectomia abdômino-endoanal com anastomose colorretal manual; RSA-ACRMe: retossigmoidectomia abdominal com anastomose colorretal mecânica; RCT-ACAMe: retocolectomia com anastomose cólon-anal mecânica; AAP: amputação abdominoperineal; PCT: proctocolectomia total. 
Tabela 3 - Cirurgias realizadas (4 tipos básicos de cirurgias: ressecção local, ressecções com anastomose manual, ressecções com anastomose mecânica e amputação abdominoperineal) em 380 pacientes portadores de câncer no reto.

\begin{tabular}{lrc}
\hline Cirurgias & $\mathbf{N}^{\mathbf{0}}$ & $\mathbf{\%}$ \\
\hline RL & 27 & 7,1 \\
Ressecções com anastomoses manuais & 133 & 35,0 \\
Ressecções com anastomoses mecânicas & 39 & 10,3 \\
Amputação com colostomia (135) e ileostomia (4) & 139 & 36,5 \\
Total de TU ressecados & 338 & 88,9 \\
Total de TU não ressecados & 35 & 9,2 \\
Total de pacientes operados & $\mathbf{3 7 3}$ & $\mathbf{9 8 , 1}$ \\
& & 98,1 \\
Total de pacientes operados & 373 & 1,9 \\
Total de pacientes não operados & 7 & $\mathbf{1 0 0 , 0}$ \\
Total de pacientes com CR & $\mathbf{3 8 0}$ & \\
\hline
\end{tabular}

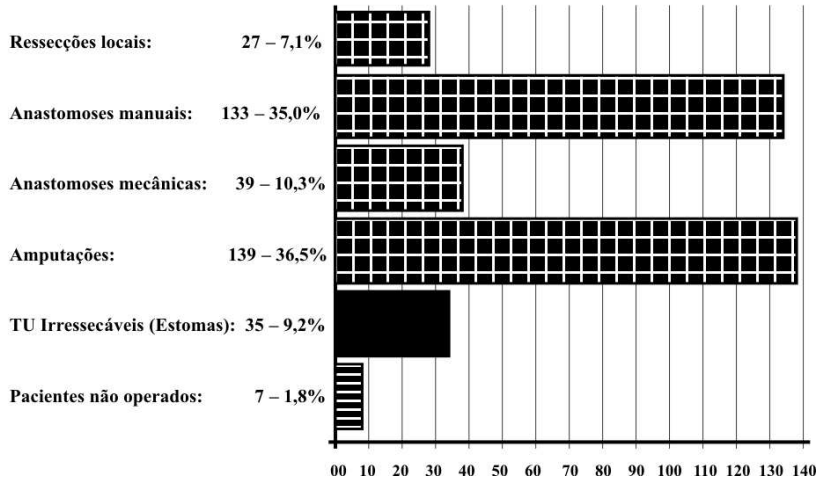

Figura 3 - Cirurgias realizadas (4 tipos básicos de cirurgias: ressecção local, ressecções com anastomose manual, ressecções com anastomose mecânica e amputação abdominoperineal) em 380 pacientes portadores de câncer no reto.

ressecções com anastomoses manuais (12,0\%), e em 5 de 39 pacientes submetidos a ressecções com anastomoses mecânicas (12,8\%). Dos 16 estomas executados em ressecções com anastomoses manuais três foram em 104 casos de retossigmoidectomia abdominal com anastomose colorretal manual (2,9\%), seis foram em 22 casos de retocolectomia total com anastomose ileorretal manual $(27,3 \%)$, e sete nos sete casos de retossigmoidectomia abdômino-endoanal com anastomose colorretal manual (100,0\%) (tabela 4).

Dos cinco estomas executados em 39 ressecções com anastomoses mecânicas $(12,8 \%)$ uma foi em 26 casos de retossigmoidectomia abdominal com anastomose colorretal manual $(3,8 \%)$ e quatro foram em 13 casos de retocolectomia total com anastomose cólon-anal mecânica (30,7\%). Dos 174 estomas definitivos 135 foram realizados em 135 pacientes submetidos à amputação abdominoperineal, quatro em quatro pacientes submetidos à proctocolectomia total com ileostomia e 35 em 35 pacientes laparotomizados e com tumores irressecáveis (tabela 4).

Sinopse dos estomas confeccionados em cirurgias randomizadas para abordagem de 380 pacientes portadores de câncer no reto:

Não houve estomas em quaisquer das 27 ressecções locais de câncer retal. Foram confeccionados 21 estomas em 172 pacientes submetidos à retossigmoidectomia abdominal $(12,2 \%)$, englobando anastomoses manuais (16 estomas em 133 casos) e mecânicas (cinco estomas em 39 casos). Foram confeccionados 174 estomas nos 135 pacientes submetidos à amputação abdominoperineal, nos quatro submetidos à proctocolectomia total e nos 35 pacientes com tumores irressecáveis (tabela 5 e figura 4).

Modalidades de estomas confeccionados em 373 cirurgias para abordagem de 380 pacientes portadores de câncer no reto, de acordo com sua permanência:

Dos 21 estomas temporários feitos em 373 pacientes operados de câncer retal $(5,6 \%)$ nove $(2,4 \%)$ foram colostomias em alça e doze ileostomias em alça $(3,2 \%)$. Dos 174 estomas definitivos realizados nos 373 pacientes portadores de câncer retal $(46,7 \%) 146$ foram colostomias terminais, quatro foram ileostomias 
Tabela 4 - Distribuição dos 195 estomas confeccionados relacionados com as 373 cirurgias realizadas em 380 pacientes portadores de câncer retal.

\begin{tabular}{|c|c|c|c|c|}
\hline \multirow[t]{2}{*}{ Cirurgias } & \multicolumn{2}{|c|}{ Cirurgias } & \multicolumn{2}{|l|}{ Estomas } \\
\hline & $\mathbf{N}$ & $\mathbf{N}$ & & $\%$ \\
\hline RL & 27 & $\mathbf{0}$ & & $\mathbf{0}$ \\
\hline \multicolumn{5}{|c|}{ Preservação esfincteriana } \\
\hline RSA-ACRMa & 104 & 3 & & 2,9 \\
\hline RCT-AIRMa & 22 & 6 & & 27,3 \\
\hline RSAEA-ACRMa & 7 & 7 & & 100,0 \\
\hline Anastomose manual & 133 & 16 & & 12,0 \\
\hline RSA-ACRMe & 26 & 1 & & 3,8 \\
\hline RC-ACAMe & 13 & 4 & & 30,7 \\
\hline Anastomose mecânica & 39 & 5 & & 12,8 \\
\hline \multirow[t]{3}{*}{ Estomas temporários } & 172 & 21 & & 12,2 \\
\hline & 373 & 21 & & 5,7 \\
\hline & 195 & 21 & & 10,8 \\
\hline AAP & 135 & 135 & $77,6: \quad 174$ & - 9,4\%: 373 \\
\hline PCT - I & 4 & 4 & $2,3 \%: 174$ & $-1,1 \%: 373$ \\
\hline TU-I - C & 35 & 35 & $20,1 \%: 174$ & $-9,4 \%: 373$ \\
\hline \multirow[t]{3}{*}{ N Estomas definitivos } & 174 & 174 & & 100,0 \\
\hline & 373 & 174 & & 46,6 \\
\hline & 195 & 174 & & 89,2 \\
\hline N Total estomas & 373 & 195 & & 52,3 \\
\hline
\end{tabular}

$R L$ : resseção local;

RSA-ACRMa: retossigmoidectomia abdominal com anastomose colorretal manual;

RCT-AIRMa: retocolectomia total com anastomose ileorretal manual;

RSAEA-ACRMa: retossigmoidectomia abdômino-endoanal com anastomose colorretal manual;

RSA-ACRMe: retossigmoidectomia abdominal com anastomose colorretal mecânica;

$R C$-ACAMe: retocolectomia com anastomose cólon-anal mecânica;

AAP: amputação abdominoperineal à Miles com colostomia definiiva;

PCT - I: proctocolectomia total com ileostomia.

terminais $(1,1 \%), 21$ foram colostomias em alça $(5,7 \%)$ e três foram colostomias duplas $(0,8 \%)$. Na totalização, dos 195 estomas realizados em 373 pacientes operados por câncer retal $(52,3 \%) 146$ foram colostomias terminais $(39,1 \%)$, quatro foram ileostomias terminais $(1,1 \%), 30$ foram colostomias em alça $(8,0 \%)$, três foram colostomias duplas $(0,8 \%)$ e doze foram ileostomias em alça $(3,3 \%)$ (tabela 6 e figura 5).

Distribuição das várias modalidades dos estomas confeccionados nas várias técnicas cirúrgicas realizadas na abordagem cirúrgica de 380 pacientes portadores de câncer no reto:

Dos 21 estomas temporários feitos em 172 pacientes submetidos à ressecções com preservação dos esfincteres (12,2\%), 16 foram em 133 ressecções com anastomoses manuais $(12,0 \%)$ e cinco foram em
29 ressecções com anastomoses mecânicas $(17,3 \%)$. Dos 16 casos de estomas em 133 ressecções com anastomoses manuais, oito foram colostomias em alça $(6,0 \%)$ e oito foram ileostomias em alça $(6,0 \%)$ (tabela 7). Dos cinco casos de estomas em 29 ressecções com anastomoses mecânicas uma foi colostomia em alça $(3,5 \%)$ e quatro foram ileostomia em alça $(13,8 \%)$ (tabela 7).

Dos 21 estomas temporários realizados em 172 casos de ressecções com anastomoses manuais e mecânicas $(12,2 \%)$, nove foram colostomias em alça $(5,2 \%)$ e 12 foram ileostomias em alça $(7,0 \%)$, ambas protetoras de anastomoses (tabela 7). Desses 21 estomas 16 foram em 133 ressecções com anastomoses manuais, assim distribuídos: três colostomias em alça em 104 casos de retossigmoidectomia abdominal com 
Tabela 5 - Incidência de estomas pelas cirurgias randomizadas realizadas em 373 de 380 pacientes portadores de câncer retal.

\begin{tabular}{lrrr}
\hline Parâmetros & Cirurgias & \multicolumn{2}{c}{ Estomas } \\
\cline { 2 - 4 } & $\mathbf{N}^{\mathbf{0}}$ & $\mathbf{N}$ & $\mathbf{0}$ \\
\hline RL & $\mathbf{2 7}$ & $\mathbf{0}$ & $\mathbf{0}$ \\
Ressecções com anastomoses manuais & 133 & 16 & 12 \\
Ressecções com anastomoses mecânicas & 39 & 5 & 12,8 \\
Estomas temporários & $\mathbf{1 7 2}$ & $\mathbf{2 1}$ & $\mathbf{1 2 , 2}$ \\
AAP & 135 & 135 & \\
PCT-I & 4 & 4 & \\
TUI - colostomia & 35 & 35 & \\
Estomas definitivos & $\mathbf{1 7 4}$ & $\mathbf{1 7 4}$ & $\mathbf{1 0 0 , 0}$ \\
Total cirurgias \& estomas & $\mathbf{3 7 3}$ & $\mathbf{1 9 5}$ & $\mathbf{5 2 , 3}$ \\
\hline
\end{tabular}

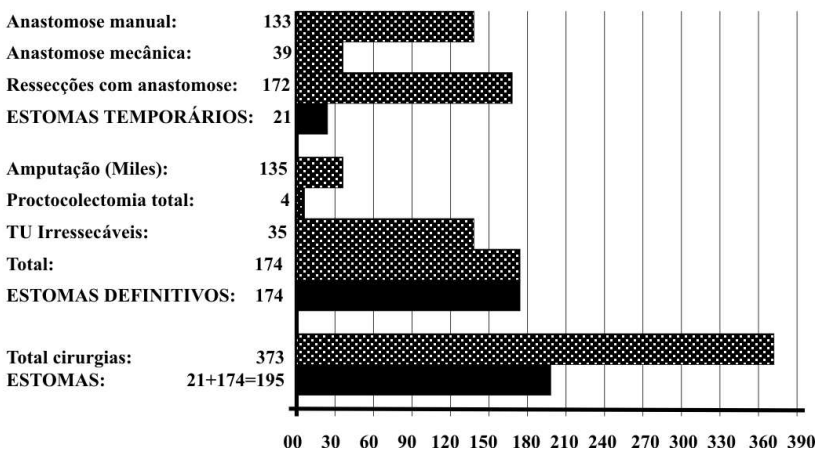

Figura 4 - Sinopse da distribuição dos 195 estomas relacionados com as 373 cirurgias realizadas em 380 pacientes portadores de câncer retal.

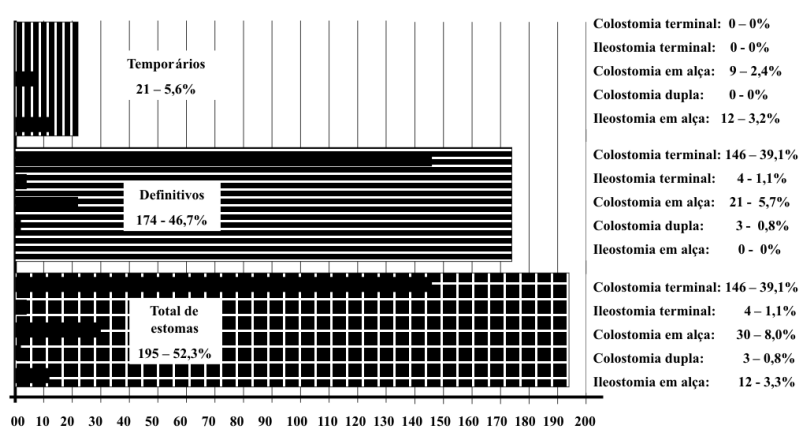

Figura 5 - Distribuição dos 174 estomas definitivos e dos 21 estomas temporários em 373 cirurgias em 380 pacientes portadores câncer do reto, pelas modalidades dos mesmos.

anastomose colorretal manual; seis ileostomias em alça em 22 casos de retocolectomia total com anastomose ileorretal manual e sete estomas em sete casos de retossigmoidectomia abdômino-endoanal com
Tabela 6 - Distribuição dos 174 estomas definitivos e dos 21 estomas temporários em 373 cirurgias em 380 pacientes portadores câncer do reto, pelas modalidades dos mesmos.

\begin{tabular}{lrc}
\hline Estomas & \multicolumn{2}{c}{ Reto (x/373 operados) } \\
\cline { 2 - 3 } & $\mathbf{N}$ & $\mathbf{\%}$ \\
\hline Estomas temporários & $\mathbf{2 1}$ & $\mathbf{5 , 6}$ \\
Colostomia terminal & 0 & 0 \\
Ileostomia terminal & 0 & 0 \\
Colostomia em alça & 9 & 2,4 \\
Colostomia dupla & 0 & 0 \\
Ileostomia em alça & 12 & 3,2 \\
Estomas definitivos & $\mathbf{1 7 4}$ & $\mathbf{4 6 , 7}$ \\
Colostomia terminal & 146 & 39,1 \\
Ileostomia terminal & 4 & 1,1 \\
Colostomia em alça & 21 & 5,7 \\
Colostomia dupla & 3 & 0,8 \\
Ileostomia em alça & 0 & 0 \\
Total de estomas & $\mathbf{1 9 5}$ & $\mathbf{5 2 , 3}$ \\
Colostomia terminal & 146 & 39,1 \\
Ileostomia terminal & 4 & 1,1 \\
Colostomia em alça & 30 & 8,0 \\
Colostomia dupla & 3 & 0,8 \\
Ileostomia em alça & 12 & 3,3 \\
\hline
\end{tabular}

anastomose colorretal manual (cinco colostomias em alça e duas ileostomias em alça) (tabela 6). E cinco foram estomas em 39 ressecções com anastomoses mecânicas (12,8\%), assim distribuídas: uma colostomia 
Estoma \& Câncer Retal: Revisão de 195 Estomas Realizados

em 380 Pacientes Portadores de Câncer Retal

Geraldo Magela Gomes da Cruz e Cols.
Vol. 28 em alça em 26 retossigmoidectomias abdominais com anastomose colorretal mecânica e quatro ileostomias em alça em 13 retocolectomia total com anastomose cólon-anal mecânica (tabela 7).

Nos 174 pacientes nos quais foram feitos estomas definitivos em $146(83,9 \%)$ casos foram feitos colostomias terminais, em quatro ileostomias terminais (2,3\%), em 21 colostomias em alça $(12,1 \%)$, e em três colostomia dupla (1,7\%). Dos 174 estomas definitivos realizados em 174 pacientes, em 135 (amputação abdominoperineal) a colostomia foi terminal; nos quatro pacientes submetidos à proctocolectomia total com ileostomia definitiva, a ileostomia foi terminal; e nos 35 pacientes submetidos à laparotomia com tumores irressecáveis, em 11 foi feita colostomia terminal, em 21 colostomia em alça e em três colostomia dupla (tabela 7).
Na totalização, dos 195 estomas realizados em 373 pacientes operados por câncer retal $(52,2 \%)$, foram as seguintes as modalidades de estomas confeccionados: 146 colostomias terminais $(39,1 \%)$, quatro ileostomias terminais $(1,1 \%), 30$ colostomias em alça $(8,0 \%)$, três colostomias duplas $(0,8 \%)$ e 12 ileostomias em alça $(3,2 \%)$ (tabela 7$)$.

Complicações das modalidades dos 195 estomas confeccionados relacionados com aos vários procedimentos cirúrgicos realizados em 373 pacientes operados por câncer no reto:

Houve uma incidência de complicações de 7,2\% (14 complicações em 195 estomas executados), assim distribuídos: oito complicações em 146 colostomias terminais $(5,5 \%)$, uma complicação dentre as 30 colostomias em alça $(3,3 \%)$, três complica-

Tabela 7 - Distribuição das várias modalidades dos estomas confeccionados nas várias técnicas cirúrgicas realizadas na abordagem cirúrgica de 380 pacientes portadores de câncer no reto.

\begin{tabular}{lccccccc}
\hline Cirurgias & Cirurgia & Estoma & $\begin{array}{c}\text { Colos } \\
\text { term }\end{array}$ & $\begin{array}{c}\text { Íleos } \\
\text { term }\end{array}$ & $\begin{array}{c}\text { Colos } \\
\text { alça }\end{array}$ & $\begin{array}{c}\text { Colos } \\
\text { dupla }\end{array}$ & $\begin{array}{c}\text { Ileos } \\
\text { alça }\end{array}$ \\
\hline Ressecções locais & $\mathbf{2 7}$ & $\mathbf{0}$ & $\mathbf{0}$ & $\mathbf{0}$ & $\mathbf{0}$ & $\mathbf{0}$ & $\mathbf{0}$ \\
RSA-ACRMa & 104 & 3 & 0 & 0 & 3 & 0 & 0 \\
RCT-AIRMa & 22 & 6 & 0 & 0 & 0 & 0 & 6 \\
RSAEA-ACRMa & 7 & 7 & 0 & 0 & 5 & 0 & 2 \\
N anastomose manual & $\mathbf{1 3 3}$ & $\mathbf{1 6}$ & $\mathbf{0}$ & $\mathbf{0}$ & $\mathbf{8}$ & $\mathbf{0}$ & $\mathbf{8}$ \\
\% anastomose manual & & $\mathbf{1 2 , 0}$ & $\mathbf{0}$ & $\mathbf{0}$ & $\mathbf{6 , 0}$ & $\mathbf{0}$ & $\mathbf{6 , 0}$ \\
RSA-ACRMe & 26 & 1 & 0 & 0 & 1 & 0 & 0 \\
RC-ACAMe & 13 & 4 & 0 & 0 & 0 & 0 & 4 \\
N anastomose mecânica & $\mathbf{3 9}$ & $\mathbf{5}$ & $\mathbf{0}$ & $\mathbf{0}$ & $\mathbf{1}$ & $\mathbf{0}$ & $\mathbf{4}$ \\
\% anastomose mecânica & & 12,8 & 0 & 0 & 2,6 & 0 & 10,2 \\
N Estoma temporário & $\mathbf{1 7 2}$ & $\mathbf{2 1}$ & $\mathbf{0}$ & $\mathbf{0}$ & $\mathbf{9}$ & $\mathbf{0}$ & $\mathbf{1 2}$ \\
\% estoma temporário & & 12,2 & 0 & 0 & 5,2 & 0 & 7,0 \\
AAP - Miles & 135 & 135 & 135 & 0 & 0 & 0 & 0 \\
PCT - Ileostomia & 4 & 4 & 0 & 4 & 0 & 0 & 0 \\
TU-I - Colostomia & 35 & 35 & 11 & 0 & 21 & 3 & 0 \\
N Estoma definitivo & $\mathbf{1 7 4}$ & $\mathbf{1 7 4}$ & $\mathbf{1 4 6}$ & $\mathbf{4}$ & $\mathbf{2 1}$ & $\mathbf{3}$ & $\mathbf{0}$ \\
\% estoma definitivo & & 100 & 83,9 & 2,3 & 12,1 & 1,7 & 0 \\
N Total estomas & $\mathbf{3 7 3}$ & $\mathbf{1 9 5}$ & $\mathbf{1 4 6}$ & $\mathbf{4}$ & $\mathbf{3 0}$ & $\mathbf{3}$ & $\mathbf{1 2}$ \\
\% Total estomas & & $\mathbf{5 2 , 2}$ & $\mathbf{3 9 , 1}$ & $\mathbf{1 , 1}$ & $\mathbf{8 , 0}$ & $\mathbf{0 , 8}$ & $\mathbf{3 , 2}$ \\
\hline
\end{tabular}

$R L$ : resseção local;

RSA-ACRMa: retossigmoidectomia abdominal com anastomose colorretal manual;

RCT-AIRMa: retocolectomia total com anastomose ileorretal manual;

RSAEA-ACRMa: retossigmoidectomia abdômino-endoanal com anastomose colorretal manual;

RSA-ACRMe: retossigmoidectomia abdominal com anastomose colorretal mecânica;

$R C$-ACAMe: retocolectomia com anastomose cólon-anal mecânica;

AAP: amputação abdominoperineal à Miles com colostomia definiiva;

PCT - I: proctocolectomia total com ileostomia. 
Estoma \& Câncer Retal: Revisão de 195 Estomas Realizados

em 380 Pacientes Portadores de Câncer Retal

Geraldo Magela Gomes da Cruz e Cols.
Vol. 28 ção nas três colostomias duplas $(100,0 \%)$, duas complicações em 12 ileostomias am alça $(16,7 \%)$ e nenhuma complicação entre as quatro ileostomias terminais (tabela 8 e figura 6).

Foram as seguintes as oito complicações das 146 colostomias terminais $(5,5 \%)$ : uma necrose, dois desabamentos, três estenoses tubulares, um prolapso e uma hérnia paracolostômica; nenhuma complicação entre as quatro ileostomias terminais (0\%); a única complicação das 30 colostomias em alça $(3,3 \%)$ foi necrose; das três complicações apresentadas pelas três colostomias duplas, uma foi estenose tubular e duas foram prolapsos; e das duas complicações das 12 ileostomias em alça (16,7\%)uma foi necrose e uma foi prolapso (tabela 9 e figura 7).

\section{DISCUSSÃO}

$\mathrm{O}$ câncer colorretal insere-se entre os quatro cânceres mais frequientes no Brasil, excluindo-se os tumores de pele, sendo a quinta causa de morte entre as malignidades. Ocupa os terceiro e quarto lugares em incidência, nas mulheres e nos homens, respectivamente. Estimam-se 20.000 casos novos por ano em nosso país ${ }^{1,2}$.

Localiza-se, predominantemente, no reto (43 $52,5 \%$ dos casos), seguido pelo cólon sigmóide (21,6 $25 \%$ ) e outros segmentos colônicos ${ }^{1,2,3,4}$. Estes dados estão em correspondência com nossa casuística, que mostrou 41,2\% de tumores localizados no reto e 53,1\% nos cólons.

O tratamento do câncer de reto é multidisciplinar, envolvendo, geralmente, o coloproctologista, o oncologista e o radioterapeuta. A cirurgia continua sendo a melhor opção terapêutica quando se pretende tratamento curativo, tendo ao seu lado a radioterapia e a quimioterapia ${ }^{4}$.

Com a introdução da excisão total do mesorreto (Heald et al, 1985) ${ }^{13}$ foi possível a melhoria da qualidade de vida dos pacientes, devido à preservação dos esfíncteres anais. Entretanto, os casos de invasão da musculatura esfincteriana, os tumores indiferenciados localizados a menos de dois $\mathrm{cm}$ da linha pectínea e os casos de dificuldade técnica (obesos, homens com pelve muito estreita, tumores muito volumosos) indicam a amputação abdominoperineal.

No presente estudo, $45,3 \%$ dos pacientes foram tratados por retossigmoidectomia abdominal com anastomose colorretal e $36,6 \%$ por amputação abdominoperineal. Outros trabalhos mostraram resultados semelhantes: Saad-Rossne et al ${ }^{2}$ demonstraram $57 \%$ de ressecções com anastomoses e $25 \%$ de amputações abdominoperineais nos seus pacientes porta-

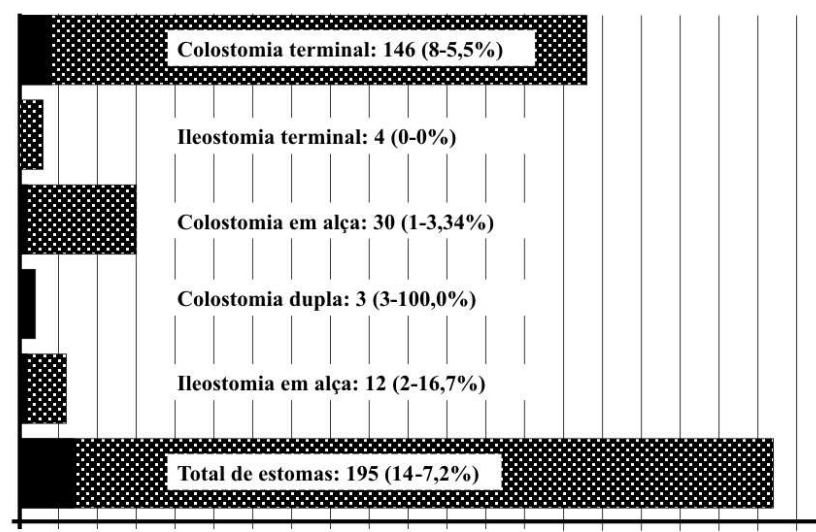

$\begin{array}{lllllllllllllllllllll}00 & 10 & 20 & 30 & 40 & 50 & 60 & 70 & 80 & 90 & 100 & 110 & 120 & 130 & 140 & 150 & 160 & 170 & 180 & 190 & 200\end{array}$

Figura 6 - Incidência de complicações das várias modalidades dos 195 estomas confeccionados relacionados com os vários procedimentos cirúrgicos realizados em 373 pacientes operados de câncer no reto.

Tabela 8 - Incidência de complicações das várias modalidades dos 195 estomas confeccionados relacionados com os vários procedimentos cirúrgicos realizados em 373 pacientes operados de câncer no reto.

\begin{tabular}{lcccc}
\hline Estomas & \multicolumn{2}{c}{ Estomas $\mathbf{x} / \mathbf{3 7 3}$ operados } & \multicolumn{2}{c}{ Complicações $\mathbf{x} / \mathbf{1 9 5}$ estomas } \\
\cline { 2 - 5 } & $\mathbf{N}$ & $\mathbf{\%}$ & $\mathbf{N}$ & $\mathbf{\%}$ \\
\hline Colostomia terminal & 146 & 39,1 & 8 & 5,5 \\
Ileostomia terminal & 4 & 1,1 & 0 & 0 \\
Colostomia em alça & 30 & 8,0 & 1 & 3,3 \\
Colostomia dupla & 3 & 0,8 & 3 & $100, \mathrm{o}$ \\
Ileostomia em alça & 12 & 3,3 & 2 & 16,7 \\
Total de estomas & $\mathbf{1 9 5}$ & $\mathbf{5 2 , 3}$ & $\mathbf{1 4}$ & $\mathbf{7 , 2}$ \\
\hline
\end{tabular}


Tabela 9 - Tipos de complicações (14) em 195 estomas em 373 pacientes operados de Câncer retal.

\begin{tabular}{|c|c|c|c|c|c|c|c|}
\hline \multirow[t]{2}{*}{ Tipos de estomas } & \multicolumn{2}{|c|}{$\begin{array}{l}\text { Complicações } \\
\text { em } 195 \text { estomas }\end{array}$} & \multicolumn{5}{|c|}{$\begin{array}{l}\text { Tipos de complicações (14) em } 195 \text { estomas em } \\
373 \text { pacientes operados de Câncer retal }\end{array}$} \\
\hline & $\mathbf{N}$ & $\%$ & Necrose & Desabamento & $\begin{array}{c}\text { Estenose } \\
\text { Tubular }\end{array}$ & Prolapso & $\begin{array}{l}\text { Hérnia } \\
\text { Paracol }\end{array}$ \\
\hline Colostomia terminal & $146-8$ & 5,5 & 1 & 2 & 3 & 1 & 1 \\
\hline Ileostomia terminal & $4-0$ & 0 & 0 & 0 & 0 & 0 & 0 \\
\hline Colostomia em alça & $30-1$ & 3,3 & 1 & 0 & 0 & 0 & 0 \\
\hline Colostomia dupla & $3-3$ & 100,0 & 0 & 0 & 1 & 2 & 0 \\
\hline Ileostomia em alça & $12-2$ & 16,7 & 1 & 0 & 0 & 1 & 0 \\
\hline Total de estomas & 14 & 7,2 & 3 & 2 & 4 & 4 & 1 \\
\hline
\end{tabular}

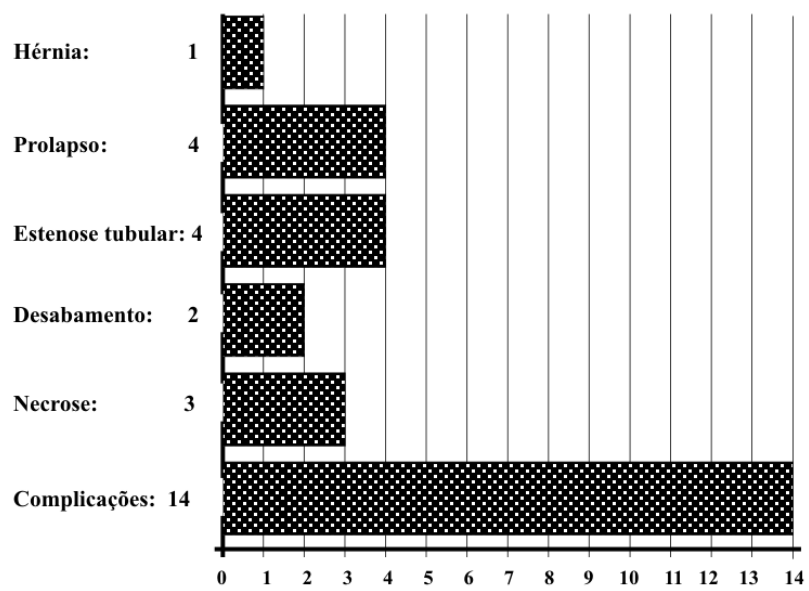

Figura 7 - Tipos de complicações (14) em 195 estomas em 373 pacientes operados de CR.

dores de câncer colorretal ${ }^{2}$. Pinho et al $(2006)^{3}$ tiveram $45,3 \%$ de retossigmoidectomias e $27 \%$ de amputações abdominoperineais. ${ }^{3}$ A maior porcentagem de amputações em nosso estudo deve-se ao fato de grande quantidade dos nossos doentes terem sido operados antes do conceito atual da excisão total do mesorreto, portanto, mais pacientes tinham seus esfíncteres ressecados.

$7,1 \%$ dos nossos casos foram tratados por ressecção local, incidência semelhante à encontrada na literatura. Nos EUA, até 5\% dos pacientes são tratados por este método.

A confecção de estomas nas cirurgias de câncer retal é realizada em pacientes submetidos à amputação abdominoperineal, por motivos óbvios, e naqueles pacientes submetidos às ressecções com preservação do esfíncter, quando as anastomoses são muito baixas ou por imperfeições técnicas. O estoma prote- tor ajuda a minimizar as graves complicações decorrentes de uma fístula anastomótica ${ }^{5}$.

No presente trabalho, $52,3 \%$ dos pacientes receberam algum tipo de estoma, sendo $46,6 \%$ definitivos e 5,6\% temporários. Os estomas definitivos foram realizados em todos os pacientes submetidos à amputação abdominoperineal, proctocolectomia total com ileostomia e nos tumores irressecáveis. Meyerhardt et al (2004) ${ }^{6}$ mostraram, em um estudo multicêntrico com 1330 pacientes, que a proporção de ressecções abdominoperineais e a conseqüente realização de colostomias definitivas no tratamento do câncer retal está relacionada ao volume de casos operados em cada serviço, sendo $46,4 \%$ nos serviços com baixo volume cirúrgico, $41,3 \%$ naqueles com volume intermediário e $31,8 \%$ naqueles com alto volume. ${ }^{6}$ Já a incidência de estomas temporários varia na literatura entre $30,7 \%$ e $41 \%$ 7,8,9.

A taxa de complicação dos estomas em nossa casuística foi de 7,2\%, taxa esta impossível de ser comparada às taxas relatadas na literatura, uma vez que, em alguns estudos são levados em consideração complicações mínimas (infecções urinárias, incontinência urinária de alguns dias, incontinência fecal de alguns dias, pequenos abscessos de parede abdominal, dentre outros). Exatamente por este motivo algumas taxas de morbilidade variam em torno de $10 \%$ e outras em torno de até $70 \%{ }^{10,11,12}$.

\section{CONCLUSÕES}

1. A incidência de câncer colorretal foi de $3,8 \%$ em atendimento coloproctológico.

2. A relação de CR no intestino grosso foi de $41,2 \%$ (380 casos). 
Estoma \& Câncer Retal: Revisão de 195 Estomas Realizados em 380 Pacientes Portadores de Câncer Retal Geraldo Magela Gomes da Cruz e Cols.
Vol. 28
3. A operabilidade dos pacientes foi de $98,2 \%$

4. A ressecabilidade dos tumores foi de $91,8 \%$

5. As cirurgias mais realizadas no $\mathrm{CR}$ foram a retossigmoidectomia $(45,3 \%)$ e a amputação abdominoperineal $(35,5 \%)$.

6. A incidência de estomas foi de 52,3\% (195 casos), a maioria definitiva (174/195, 89,2\%).

7. Os estomas temporários mais comuns foram as ileostomias em alça (12 casos, 7,0\%), e a principal causa foi a proteção de anastomose (16 casos).

8. Os estomas definitivos mais comuns foram as colostomias terminais (146 casos, 83,9\%), sendo a amputação abdominoperineal a principal causa (135 casos).

9. A incidência de complicações foi de 7,2\% (14 em 195 estomas), mais comuns nas colostomias duplas (100,0\% em três casos).

10. As principais complicações foram as estenoses (4 casos) e os prolapsos (4 casos).

\begin{abstract}
The aim of this report is to analyze 380 patients with rectal cancer with special concern to the 195 stomas carried out with particular reference to temporary and definitive stomas as well as several surgical techniques used and their indications and complications. Three hundred seventy three out of 380 patients underwent surgery $(98.2 \%)$ being 373 tumors resected $(\mathbf{9 1 . 8 \%})$. As far as surgical technique is concerned abdominal rectosigmoidectomy was carried out in 172 of 338 patients who had their tumors removed, being 133 with hand anastomoses (35.0) and 39 with stapled anastomoses (10.3\%). Miles procedure was made in $135(35.5 \%)$, local excision in $27(7.1 \%)$ and proctocolectomy and end ileostomy in four patients $(1.0 \%)$. One hundred ninety five of 373 patients who underwent surgical treatment had stomas created in their abdomen (52.3\%), being 174 definitive (46.6\%) and 21 temporary $(5.7 \%)$. So 174 of the 195 stomas carried out were definitive $(89.2 \%)$ and 21 temporary $(10.8 \%)$. One hundred and forty six of 195 stomas were end colostomies (39.1\%), four end ileostomies (1.1\%), 30 loop colostomies $(8.0 \%)$, three double colostomies $(\mathbf{0 . 8 \%})$ and 12 loop ileostomies (3.2\%). As far as 21 temporary stomas performed in 172 cases of local excision and abdominal rectosigmoidectomy $(\mathbf{1 2 . 2 \%})$ are concerned nine were loop colostomy $(5.2 \%)$ and 12 loop ileostomy (7.0\%). Sixteen of 21 temporary stomas were made in 133 cases of hand anastomoses, being eight loop colostomies (6.0\%) and eight loop ileostomies (6.0\%); and five in 39 cases of stapled anastomoses, being one loop colostomy (2.6\%) and four loop ileostomies $(\mathbf{1 0 . 2 \%})$. And as far as 174 definitive stomas are concerned they were performed in 135 patients undergoing Miles procedure, four in rectocolectomy and 35 in non resectable tumors. One hundred forty six stomas were end colostomy (83.9\%), four end ileostomy (2.3\%), 21 loop colostomy $(\mathbf{1 2 . 1 \%})$ and three double colostomy $(1.7 \%)$. So Miles procedure corresponded to $100.0 \%$ of end colostomy (135 cases), as well as four cases of proctocolectomy and 35 cases of acute abdomen, totalizing 174 cases of definitive stomas. Eleven of 35 stomas for non resectable tumors were end colostomy, 21 were loop colostomy and three double colostomy. The incidence of stoma complications were $7.2 \%$ (14 cases in 195 stomas): eight complications in 146 end colostomies (5.5\%) (one necrosis, two collapses, three stenosis, one prolapse and one paracolostomic hernia); one in 30 loop colostomies (3.3\%) (one necrosis); three in three double colostomies (100.0\%) (one stenosis and two prolapses); two in 12 loop ileostomies (16.7\%) (one necrosis and one prolapse); and no complications in four end ileostomies.
\end{abstract}

\section{REFERÊNCIAS}

1. Malheiros APR, Teixeira MG, Habr-Gama A, Alcântara PSM. Resultados do tratamento cirúrgico do câncer colo-retal em doentes de idade até 64 anos e de 65 anos ou mais. Rev bras Coloproct, 2005; 25(2): 128-136.

2. Saad-Rossne R, Prado RG, Bakonyi Neto A, Lopes OS, Nascimento SN, Santos CRV, Pracucho EM, Chaves FRP, Ioriatti ES, Siqueira JM. Estudo retrospectivo de pacientes portadores de câncer colorretal atendidos na Faculdade de Medicina de Botucatu no período de 2000-2004. Rev bras Coloproct, 2005; 25(1): 31-37.
3. Pinho MSL, Ferreira LC, Kleinubing Jr H. Tratamento cirúrgico do câncer colorretal: resultados a longo prazo e análise da qualidade. Rev bras Coloproct, 2006; 26(4): 422-429.

4. Cruz GMG, Ferreira RMRS, Neves PM. Cirurgias para câncer retal - estudo retrospectivo de 380 pacientes submetidos à cirurgia para câncer retal, ao longo de quatro décadas. Rev bras Coloproct, 2005; 25(4): 309-331.

5. Giuliani D, Willemsen P, Van Est F, Vanderveken M. A defunctioning stoma in the treatment of lower third rectal carcinoma. Acta Chir Belg, 2006; 106(1):40-43.

6. Meyerhardt JA, Topper JE, Niedzwiecki D, Hollis Dr, Schrag $\mathrm{D}$, Ayanian JZ et al. Impact of hospital procedure volume on 
surgical operation and long-term outcomes in high-risk curatively resected rectal cancer: findings from the Intergroup 0114 Study. J Clin Oncol, 2004; 22(1): 166-174.

7. Marusch F, Koch A, Schmidt U, Geibetaler S, Dralle H, Saeger HD, Wolff S, Nestler G, Pross M, Gastinger I, Lippert H. Value of a protective stoma in low anterior resections for rectal cancer. Dis Colon Rectum, 2002; 45(9): 1164-71.

8. Gastinger I, Marusch F, Steinert R, Wolff S, Koeckerling F, Lippert H; Working Group 'Colon/Rectum Carcinoma'. Protective defunctioning stoma in low anterior resection for rectal carcinoma. Br J Surg, 2005; 92(9): 1137-42.

9. Poon RT, Chu KW, Ho JW, Chan CW, Law WL, Wong J. Prospective evaluation of selective defunctioning stoma for low anterior resection with total mesorectal excision. World J Surg, 1999; 23(5): 463-7; discussion 467-8.

10. Robertson I, Leung F, Hughes D, Spiers M, Donnelly L, Mackenzie I, Macdonald A. Prospective analysis of stomarelated complications. Colorectal Dis, 2005; 7(3): 279-285.
11. Thalheimer A, Bueter M, Kortuem M, Thiede A, Meyer D. Morbidity of temporary loop ileostomy in patients with colorectal câncer. Dis Colon Rectum, 2006; 49(70: 1011-7.

12. Mahjoubi B, Moghimi A, Mirzaei R, Bijari A. Evaluation of the end colostomy complications and the risk factors influencing them in Iranian patients. Colorectal Dis, 2005; 7(6): 582-7.

13. Heald RJ, Ryall RDH. Recurrence and survival after total mesorectal excision for rectal cancer. Lancet 1986;1:1479-1482.

\section{Endereço para correspondência:}

GERALDO MAGELA GOMES DA CRUZ

Rua Rio de Janeiro, 2017, ap. 1401 - Lourdes

Belo Horizonte - Minas Gerais

CEP: 30160-042 